\title{
Bearing Capacity of Spatially Random Rock Masses Obeying Hoek-Brown Failure Criterion
}

\author{
Tamara AL-BITTAR ${ }^{\mathrm{a}}$, Michael MICHAEL ${ }^{\mathrm{b}}$ and Abdul-Hamid SOUBRA ${ }^{\mathrm{b}}$ \\ ${ }^{a}$ Lebanese University, Kobe, North Lebanon \\ ${ }^{\mathrm{b}}$ University of Nantes, France
}

\begin{abstract}
In this paper, a probabilistic analysis is presented to compute the ultimate bearing capacity of a strip footing resting on a spatially varying rock mass. The rock is assumed to follow the generalized Hoek-Brown failure criterion. The uniaxial compressive strength of the intact rock $\left(\sigma_{c}\right)$ was considered as a random field and the Geological Strength Index $(G S I)$ was modeled as a random variable. The deterministic model was based on numerical simulations. The uncertainty propagation methodology employed in the analysis makes use of a non-intrusive approach to build up a sparse polynomial chaos expansion for the system response. The probabilistic numerical results were presented in the case of a weightless rock mass. The variability of the ultimate bearing capacity was found to decrease with the decrease in the autocorrelation distance. Sobol indices have shown that for the very large values of the autocorrelation distance, the variability of the ultimate bearing capacity is mainly due to $\sigma_{c}$; however, in the case of very small values of the autocorrelation distance, GSI is the most weighed variable.
\end{abstract}

Keywords. Rock mechanics, Hoek-Brown failure criterion, bearing capacity, probabilistic analysis, spatial variability, sparse polynomial chaos expansion.

\section{Introduction}

The analysis and design of a strip footing resting on a rock mass obeying Hoek-Brown (HB) failure criterion are generally based on deterministic approaches. In this paper, the behavior of a strip footing resting on a HB rock mass is studied using a probabilistic approach. Our aim is to determine the probabilistic ultimate bearing capacity of a strip footing resting on a spatially varying rock mass and subjected to a vertical load. The rock mass is assumed to follow the generalized HB failure criterion (Hoek and Brown, 1980; Hoek et al., 2002). This criterion is characterized by four parameters (i) the geological strength index (GSI), (ii) the uniaxial compressive strength of the intact rock $\left(\sigma_{c}\right)$, (iii) the intact rock material constant $\left(m_{i}\right)$ and (iv) the disturbance coefficient (D). Mao et al. (2012) have modeled these four parameters as random variables and have performed a probabilistic analysis of the ultimate bearing capacity of foundations. These authors have shown that the variability of the ultimate bearing capacity is mainly due to the uniaxial compressive strength of the intact rock $\left(\sigma_{c}\right)$ and the geological strength index $(G S I)$. Based on this study, only these two parameters were considered herein as uncertain. The rock uniaxial compressive strength of the intact rock $\left(\sigma_{c}\right)$ was considered as a log-normal random field characterized by a square exponential autocorrelation function. The Expansion Optimal Linear Estimation (EOLE) method proposed by $\mathrm{Li}$ and Der Kiureghian (1993) was used to dicretize this random field. As for GSI, this parameter characterizes the overall rock mass condition and it does not represent a precise physical parameter varying in space. Thus, it cannot be modeled as a random field and will be treated herein as a random variable.

As for the probabilistic method of analysis, the classical Monte Carlo Simulation (MCS) methodology is generally used when dealing with random fields (Ching et al., 2011). In spite of being a rigorous method, MCS requires a large number of calls to the deterministic model. This is not convenient in the case where a computationally-expensive (finite element or finite difference) deterministic model is used. This paper makes use of an efficient probabilistic approach which significantly reduces the number of calls of the deterministic model. The Sparse 
Polynomial Chaos Expansion (SPCE) methodology is proposed in this regard.

The paper is organized as follows: The next section aims at presenting the deterministic model used for the computation of the ultimate bearing capacity. It is followed by a presentation of the probabilistic method. Finally, the probabilistic numerical results are presented and discussed. The paper ends with a conclusion.

\section{Deterministic Model}

The Hoek-Brown failure criterion only deals with intact rocks or heavily jointed rock masses. A heavily jointed rock mass involves sufficiently dense and randomly distributed joints so that in the scale of the problem, it can be regarded as an isotropic assembly of interlocking particles. Consequently, rocks with few discontinuities cannot be considered in this framework. The HB failure criterion can be described by the following equation (Hoek et al., 2002):

$\sigma_{1}-\sigma_{3}=\sigma_{c}\left[m\left(\sigma_{3} / \sigma_{c}\right)+s\right]^{n}$

where $\sigma_{1}$ and $\sigma_{3}$ are respectively the major and minor principal stresses at failure and $\sigma_{c}$ is the uniaxial compressive stress of the rock at failure. The parameters $m, s$ and $n$ are given by the following equations:

$$
\begin{aligned}
& m=m_{i} \cdot e^{\left(\frac{G S I-100}{28-14 D}\right)}, \quad s=e^{\left(\frac{G S I-100}{9-3 D}\right)}, \\
& n=(1 / 2)+(1 / 6)\left[e^{-G S I / 15}-e^{-20 / 3}\right]
\end{aligned}
$$

In these equations, the geological strength index (GSI) characterizes the quality of rock. On the other hand, the parameter $m_{i}$ is the value of parameter $m$ for intact rock and can be obtained from experimental tests. It varies from 4 for very fine weak rock like claystone to 33 for coarse igneous light-colored rock like granite. Finally, $D$ is the disturbance coefficient. It varies from 0.0 for undisturbed in situ rock masses to 1.0 for very disturbed rock masses.

The deterministic model used to calculate the ultimate bearing capacity of a strip footing resting on a $\mathrm{HB}$ rock mass and subjected to a vertical load was based on the commercial numerical code FLAC $^{3 \mathrm{D}}$. A footing of breadth $\mathrm{B}=1 \mathrm{~m}$ is considered in the analysis. For this calculation, a rock mass of $20 \mathrm{~m}$ wide by $6 \mathrm{~m}$ deep was considered. The rock behavior was modeled by an elastic perfectly plastic model obeying the generalized HB failure criterion. The rigid strip footing was modeled by a prescribed uniform velocity for all the rock nodes in contact with the footing. A value of $10^{-6} \mathrm{~m} /$ time step was chosen for this velocity since a smaller value was proven to negligibly decrease the value of the ultimate bearing capacity. Notice that the ultimate bearing capacity was computed by integrating the reaction forces at the different nodes of the footing when reaching the steady state of plastic flow.

\section{Probabilistic Analysis}

First, the discretisation of the log-normal random field of $\sigma_{c}$ is briefly presented. It is followed by a brief presentation of the SPCE methodology used for the probabilistic analysis.

\subsection{Discretization of the Random Field}

Consider a 2D non-isotropic log-normal random field $Z^{L N}$ described by: (i) a log-normal marginal cumulative distribution function $F_{G}$, and (ii) a square exponential autocorrelation function $\rho_{Z}^{L N}$ $\left[(x, y),\left(x^{\prime}, y^{\prime}\right)\right]$ which gives the values of the correlation between two arbitrary points $(\mathrm{x}, \mathrm{y})$ and $\left(x^{\prime}, y^{\prime}\right)$. Notice that this function is given as follows:

$$
\rho_{z}^{L N}\left[(x, y),\left(x^{\prime}, y^{\prime}\right)\right]=\exp \left(-\left(\frac{\left|x-x^{\prime}\right|}{a_{x}}\right)^{2}-\left(\frac{\left|y-y^{\prime}\right|}{a_{y}}\right)^{2}\right)
$$

where $a_{x}$ and $a_{y}$ are the autocorrelation distances along $x$ and $y$ respectively. The EOLE method proposed by Li and Der Kiureghian (1993) to discretize a random field is used herein. In this method, one should first define a stochastic grid composed of $s$ grid points (or nodes) and determine the log-normal autocorrelation matrix $\Sigma_{\chi ; \chi}^{L N}$ which gives the correlation between each grid point of the stochastic mesh and the other grid points of this mesh using Equation (3). The 
log-normal autocorrelation matrix $\sum_{\chi ; \chi}^{L N}$ should then be transformed into the Gaussian space using the Nataf transformation (Nataf, 1962). As a result, one obtains a Gaussian autocorrelation matrix $\Sigma_{\chi ; \chi}^{G}$ that can be used to discretize the Gaussian random field $Z$ as follows:

$$
\tilde{Z}(x, y) \cong \mu_{\ln Z}+\sigma_{\ln Z} \sum_{j=1}^{N} \frac{\xi_{j}}{\sqrt{\lambda_{j}}} \cdot \phi_{j} . \Omega
$$

where $\mu_{\ln Z}$ and $\sigma_{\ln Z}$ are the mean and standard deviation values of the underlying normal distribution (i.e. $\ln (Z)) ;\left(\lambda_{j}, \phi_{j}\right)$ are the eigenvalues and eigenvectors of the Gaussian autocorrelation matrix $\Sigma_{\chi ; \chi}^{G} ; \Sigma^{G} ; \Omega$ is the correlation vector between the value of the field at an arbitrary point (x,y) and its values at the different grid points; $\xi_{j}(j=1, \ldots, N)$ is a vector of standard normal random variables; and $N$ is the number of terms (expansion order) retained in EOLE method. This number $N$ is obtained (i) by sorting the eigenvalues $\lambda_{j}(j=1, \ldots, s)$ in a descending order and (ii) by choosing the number $\mathrm{N}$ of eigenmodes that leads to a variance of the error which is smaller than a prescribed tolerance $\varepsilon$ ( $\varepsilon \approx 10 \%$ in this paper). Notice that the variance of the error for EOLE is given by $\mathrm{Li}$ and Der Kiureghian (1993) as follows:

$\operatorname{Var}[Z(x, y)-\tilde{Z}(x, y)]=\sigma^{2}-\sum_{j=1}^{N} \frac{1}{\lambda_{j}}\left(\left(\phi_{j}\right)^{T} \Omega\right)^{2}$

where $Z(x, y)$ and $\tilde{Z}(x, y)$ are respectively the exact and the approximate values of the random fields at a given point $(\mathrm{x}, \mathrm{y})$ and $\left(\phi_{j}\right)^{T}$ is the transpose of the eigenvector $\phi_{j}$. Once the Gaussian random field is obtained, it should be transformed into the log-normal space by exponentiating the approximated Gaussian random field $\tilde{Z}(x, y)$ given by Equation (4).

\subsection{Sparse Polynomial Chaos Expansion SPCE for the System Response}

In this section, one first presents the polynomial chaos expansion (PCE) and then its extension, the sparse polynomial chaos expansion (SPCE). The polynomial chaos expansion methodology allows one to replace a complex deterministic model whose uncertain input parameters are random variables by a meta-model. Thus, the random system response may be easily calculated (when performing the probabilistic analysis by MCS) using a simple analytical equation. Within the PCE methodology, the system response $\Gamma$ of a given model with $M$ random variables can be expressed by a PCE as follows:

$$
\Gamma_{P C E}(\xi)=\sum_{\beta=0}^{\infty} a_{\beta} \Psi_{\beta}(\xi) \cong \sum_{\beta=0}^{P-1} a_{\beta} \Psi_{\beta}(\xi)
$$

where $\mathrm{P}$ is the number of terms retained in the truncation scheme, $\xi=\left\{\xi_{i}\right\}_{i=1, \ldots, M}$ is a vector of $\mathrm{M}$ independent standard random variables that represent the $M$ random variables, $a_{\beta}$ are unknown coefficients to be computed and $\Psi_{\beta}$ are multivariate Hermite polynomials. These multivariate Hermite polynomials can be obtained from the product of one-dimensional Hermite polynomials as follows:

$$
\Psi_{\beta}=\prod_{i=1}^{M} H_{\alpha_{i}}\left(\xi_{i}\right)
$$

where $\alpha_{i}(i=1, \ldots, M)$ are a sequence of $M$ nonnegative integers and $H_{\alpha_{i}}($.$) is the \alpha_{i}^{\text {th }}$ onedimensional Hermite polynomial. The coefficients $a_{\beta}$ of the PCE are computed in this paper using a non-intrusive technique where the deterministic calculations are done using the finite difference software $\mathrm{FLAC}^{3 \mathrm{D}}$ treated as a black box. The most used non-intrusive method is the regression approach (Blatman and Sudret, 2010). This method is used in the present work. In fact, for a PCE of order p, only the multivariate polynomials $\Psi_{\beta}$ of degree less than or equal to $\mathrm{p}$ should be retained. This leads to a number P of the unknown PCE coefficients (see 
Equation (6)) equal to $\frac{(M+p) !}{M ! p !}$. It should be noticed that the number of the PCE coefficients to be computed grows dramatically with the size $\mathrm{M}$ of the input random vector and the PCE order p. When dealing with a random field as is the case in the present paper (and especially when considering small values of the autocorrelation distance), the discretization of the random field may lead to a significant number of random variables which makes the determination of the PCE coefficients unfeasible because of the significant increase in the number of calls of the deterministic model. To address such problem, the sparse polynomial chaos expansion developed by Blatman and Sudret 2010 is used herein. Indeed, Blatman and Sudret 2010 have shown that the number of significant terms in a PCE is relatively small since the multivariate polynomials $\Psi_{\beta}$ corresponding to high-order interaction (i.e. those resulting from the multiplication of the $H_{\alpha_{i}}$ with increasing $\alpha_{\mathrm{i}}$ values) are associated with very small values for the coefficients $a_{\beta}$. Based on this observation, these authors have proposed a so-called hyperbolic truncation scheme to determine the significant $\Psi_{\beta}$ terms. This scheme suggests that the q-norm $\|\alpha\|_{q}$ of the retained $\Psi_{\beta}$ term should be less than or equal to the order $\mathrm{p}$ of the PCE. The q-norm is given by:

$$
\|\alpha\|_{q}=\left(\sum_{i=1}^{M}\left(\alpha_{i}\right)^{q}\right)^{1 / 9}
$$

where $\mathrm{q}$ is a coefficient $(0<\mathrm{q}<1)$. In this formula, q can be chosen arbitrarily. Blatman and Sudret 2010 have shown that sufficient accuracy is obtained for $q \geq 0.5$.

The proposed SPCE methodology leads to a sparse polynomial chaos expansion that contains a small number of unknown coefficients which can be calculated from a reduced number of calls of the deterministic model with respect to the classical PCE methodology. Once the SPCE coefficients are determined, a global sensitivity analysis (GSA) based on Sobol indices can be easily performed. Notice that the first order Sobol index of a given random variable $\xi_{\mathrm{i}}$ $(\mathrm{i}=1, \ldots, \mathrm{M})$ gives the contribution of this variable to the variability of the system response. The first order Sobol index is given by Salteli et al. 2000 as follows:

$$
S\left(\xi_{i}\right)=\frac{\operatorname{Var}\left[E\left(Y \mid \xi_{i}\right)\right]}{\operatorname{Var}(Y)}
$$

where $Y$ is the system response, $E\left(Y \mid \xi_{i}\right)$ is the expectation of $Y$ conditional on a fixed value of $\xi_{i}$, and $V a r$ denotes the variance. In the present paper, the system response is represented by a SPCE. Thus, by replacing $Y$ in Equation (9) with the SPCE expression, one obtains the Sobol index as a function of the different terms of the SPCE (Sudret, 2008) as follows:

$$
S\left(\xi_{i}\right)=\frac{\sum_{\beta \in I_{i}}\left(a_{\beta}\right)^{2} E\left[\left(\Psi_{\beta}\right)^{2}\right]}{\sum_{\beta \in\left[l_{1}, I_{2}, \ldots, I_{N}\right]}\left(a_{\beta}\right)^{2} E\left[\left(\Psi_{\beta}\right)^{2}\right]}
$$

where $a_{\beta}$ are the obtained SPCE coefficients, $\Psi_{\beta}$ are the multivariate Hermite polynomials, $\mathrm{E}[$.$] is the expectation operator, and E\left[\left(\Psi_{\beta}\right)^{2}\right]$ is given by Sudret (2008) as follows:

$$
E\left(\Psi_{\beta}^{2}\right)=\prod_{i=1}^{M} \alpha_{i} !
$$

In this equation, $\alpha_{i}$ are the same sequence of $M$ non-negative integers used in Eq (7). Notice finally that $I_{i}$ which appears in the numerator of Eq (10) denotes the set of indices $\beta$ for which the corresponding $\Psi_{\beta}$ terms are only functions of the random variable $\xi_{\mathrm{i}}$ (i.e. they only contain the variable $\left.\xi_{\mathrm{i}}\right)$, and $\mathrm{I}_{\mathrm{i}}(\mathrm{i}=1, \ldots, \mathrm{M})$ in the denominator of the same equation regroup all the indices $\beta$ for which the corresponding $\Psi_{\beta}$ terms are functions of all the random variables $\xi_{\mathrm{i}}(\mathrm{i}=1$, $\ldots, \mathrm{M})$. In the present paper where both a random variable $(G S I)$ and a random field $\left(\sigma_{c}\right)$ are involved, the Sobol index of the random field $\left(\sigma_{c}\right)$ is computed as the sum of the Sobol indices of the different variables that represent this field. 


\section{Numerical Results}

The aim of this section is to present the probabilistic numerical results. The mean value and coefficient of variation of $\sigma_{c}$ (referred to in this paper as reference values) were taken as follows: $\mu_{\sigma_{c}}=10 \mathrm{MPa}, \mathrm{COV} \sigma_{\sigma_{c}}=25 \%$. On the other hand, the mean value and coefficient of variation of GSI are given as follows: $\mu_{G S I}=25, C_{G S}=10 \%$. As for the autocorrelation distance $(a)$ of the random field $\sigma_{c}$, the reference value adopted is $2 \mathrm{~m}$. Notice that the intact rock material constant $\left(m_{i}\right)$ and the disturbance coefficient $(D)$ were assumed to be deterministic. Their corresponding values were respectively $m_{i}=8$ and $\mathrm{D}=0.3$.

\subsection{Sobol Indices}

Figure 1 depicts the values of Sobol indices (for the reference case) as given by the obtained SPCE for (i) the random variable GSI and (ii) the 35 random variables representing the random field $\sigma_{c}$. The first random variable $\xi_{1}$ corresponds to GSI and its Sobol index was found to be equal to 0.66 . However, the last 35 random variables [i.e. $\xi_{\mathrm{i}}$ for $\mathrm{i}=2, \ldots, 36$ ] are those corresponding to the $\sigma_{c}$ random field. The sum of their Sobol indices gives the weight of the random field $\sigma_{c}$ in the variability of the ultimate bearing capacity. This sum was found to be equal to 0.34 .

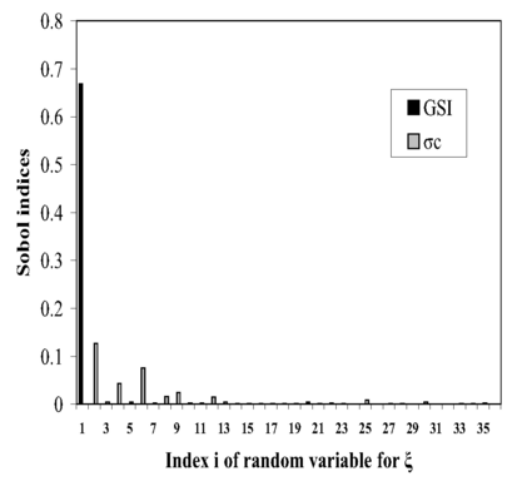

Figure 1. Sobol indices of the random variable GSI [i.e. $\xi i(i=1)]$ and the random field $\sigma c[$ i.e. $\xi i(i=2, \ldots, 36)]$

Figure 1 shows that only six random variables $\left(\xi_{2}\right.$, $\left.\xi_{4}, \xi_{6}, \xi_{8}, \xi_{9}, \xi_{12}\right)$ of the $\sigma_{c}$ random field are the most influential (they involve $89 \%$ of the variance of $\sigma_{c}$ ). This can be explained by the fact that the ultimate bearing capacity is a quantity that depends on the average distribution of the spatially-varying rock property over the entire domain and it is therefore quite insensitive to small-scale fluctuations of $\sigma_{c}$. Notice that the first eigenmodes provide the average distribution of $\sigma_{c}$ over the rock domain; however, the remaining eigenmodes give the small scale fluctuations around this average distribution.

\subsection{Effect of the Autocorrelation Distance}

Table 1 presents the first two statistical moments of the ultimate bearing capacity for different values of the autocorrelation distance of $\sigma_{c}$. This table shows that the variability of the bearing capacity decreases when the autocorrelation distance decreases. For the very large values of the autocorrelation distance (i.e. $a=100 \mathrm{~m}$ ), the coefficient of variation of the ultimate bearing capacity tends to a constant maximal value which is the value corresponding to the case of a random variable. This is because in this case the value of $\sigma_{c}$ (which is uniform within a given realization) varies within a large range corresponding to the values obtained from the PDF of $\sigma_{c}$. The decrease in the autocorrelation distance of the random field $\sigma_{c}$ from infinity to a finite value (moderate or small where $a \leq 10 \mathrm{~m}$ ) limits the correlation between the values of this random field (in a given simulation) to a finite zone which leads to several zones with different values of $\sigma_{c}$ over the entire rock mass. This means that in a single simulation, one obtains a set of weak and strong zones. The position of these zones may change from simulation to another one. This leads to a decrease in the variability of the ultimate bearing capacity because the presence of the rock mass heterogeneity will produce a somewhat close global behavior of the footing because of the averaging phenomenon over the zone of possible failure mechanism. 
Table 1. Effect of the autocorrelation distance $a$ on the statistical moments $(\mu, \sigma$,$) of the ultimate bearing capacity$

\begin{tabular}{llll}
\hline $\begin{array}{l}\text { Autocorrelation } \\
\text { distance } \mathrm{a}[\mathrm{m}]\end{array}$ & $\mu[\mathrm{MPa}]$ & $\sigma[\mathrm{MPa}]$ & $\mathrm{COV} \%$ \\
\hline 0.5 & 1.486 & 0.288 & 19.35 \\
1 & 1.459 & 0.301 & 20.88 \\
2 & 1.462 & 0.342 & 23.40 \\
5 & 1.484 & 0.408 & 27.49 \\
10 & 1.512 & 0.450 & 29.78 \\
50 & 1.557 & 0.486 & 31.41 \\
100 & 1.560 & 0.488 & 31.41 \\
Random variable & 1.560 & 0.488 & 31.41 \\
\hline
\end{tabular}

Table 1 shows that the probabilistic mean value of the ultimate bearing capacity presents a minimum when the autocorrelation distance is nearly equal to the footing breadth B (i.e. when $a$ $=1 \mathrm{~m})$. Notice that the minimal probabilistic mean was also observed by Fenton and Griffiths (2003) when considering the bearing capacity of foundations resting on a soil mass.

Finally, Figure 2 shows the effect of the autocorrelation distance on the Sobol indices of the random field $\sigma_{c}$ and the random variable GSI. The results show that for very large values of the autocorrelation distance, the variability of the ultimate bearing capacity is mainly due to $\sigma_{c}$. Similar results were obtained by Mao et al. (2012) where the uncertain parameters were modeled by random variables. On the other hand, Figure 2 shows that the decrease in the autocorrelation distance of $\sigma_{c}$ reduces its weight in the variability of the ultimate bearing capacity and increases the weight of GSI. This result can be explained by the fact that the small values of the autocorrelation distance increase the rock mass heterogeneity (i.e. one obtains a set of weak and strong zones) which will produce a somewhat close global behavior of the footing from simulation to another one because of the averaging phenomenon over the zone of possible failure mechanism. The expected decrease in the variability of the ultimate bearing capacity with the decrease in the autocorrelation distance of $\sigma_{c}$ is reflected herein by a decrease in the weight of $\sigma_{c}$ in the variability of this response.

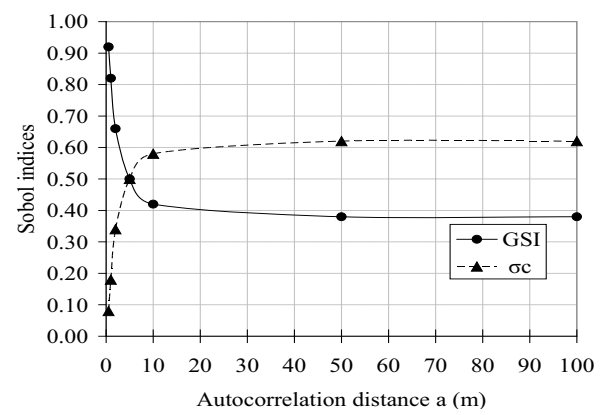

Figure 2. Influence of the autocorrelation distance $a$ on the Sobol indices of GSI and $\sigma_{c}$

\section{Conclusions}

A probabilistic analysis of a vertically loaded strip footing resting on a spatially varying rock mass has been performed to compute the ultimate bearing capacity. The rock was assumed to follow the generalized Hoek-Brown failure criterion. The uniaxial compressive strength of the intact rock $\left(\sigma_{c}\right)$ was considered as a random field and the Geological Strength Index (GSI) was modeled as a random variable. The uncertainty propagation methodology employed in the analysis makes use of a non-intrusive approach to build up a sparse polynomial chaos expansion for the system response. Also, a global sensitivity analysis based on Sobol indices was performed. The deterministic model was based on numerical simulations using FLAC ${ }^{3 \mathrm{D}}$ software. The probabilistic numerical results were presented in the case of a weightless rock mass. These results have shown that for the very large values of the autocorrelation distance, the variability of the ultimate bearing capacity is mainly due to $\sigma_{c}$; however, in the case of very small values of the autocorrelation distance, GSI is the most weighed variable. With a decrease in the autocorrelation distance of the uniaxial compressive strength of the intact rock $\left(\sigma_{c}\right)$, a smaller variability of the ultimate bearing capacity was obtained; however, the probabilistic mean value of the ultimate bearing capacity presents a minimum. This minimum was obtained when the autocorrelation distance is equal to the footing breadth B. 


\section{References}

Hoek E, Brown E. Empirical strength criterion for rock masses. J Geotech Eng Div 1980; 106(GT9):1013-35.

Hoek E, Carranze-Torres C, Corkum B. Hoek-Brown failure criterion-2002 edition. In: Proceeding of the North American rock mechanics society meeting, Toronto, July 2002. p. 267-73.

Mao N, Al-Bittar T, and Soubra AH. Probabilistic analysis and design of strip foundations resting on rocks obeying Hoek-Brown failure criterion. Int. J. Rock. Mech. Mining Sci 2012(a); 49: 45-58.

Li C. C, Der Kiureghian, A. Optimal discretization of random fields. Journal of Engineering Mechanics 1993; 119: 1136-54.

Ching J, Hu YG, Yang ZY Shiau JQ, Chen JC, Li YS. Reliability-based design for allowable bearing capacity of footings on rock masses by considering angle of distortion. Int. J. Rock. Mech. Mining Sci 2011; 48: 728-740.

Nataf A. Détermination des distributions de probabilités dont les marges sont données. Comptes Rendus de l'Académie des Sciences 1962; 225: 42-3 (in French).

Blatman G, Sudret B. An adaptive algorithm to build up sparse polynomial chaos expansions for stochastic finite element analysis. Probabilistic Engineering Mechanics 2010; 25: 183-197.

Saltelli, A., Chan, K., and Scott, E. M. Sensitivity Analysis. John wiley and sons 2000 .

Sudret, B. Global sensitivity analysis using polynomial chaos expansion. Reliability Engineering and System Safety 2008; 93: 964-979.

Fenton, GA, Griffiths DV. Bearing capacity prediction of spatially random $c-\varphi$ soils. Canadian Geotechnical Journal 2003; 40: 54-65. 